\title{
Textsorten und Textarbeit in Lehrwerken für Wirtschaftsdeutsch
}

\author{
Goranka Rocco
}

\begin{abstract}
Zusammenfassung
Im vorliegenden Beitrag geht es um die Fachsprache »Wirtschaftsdeutsch « als Lehr- und Lerngegenstand in den aktuellen Lehrwerken. Im Zentrum des Interesses stehen die schriftliche Wirtschaftskommunikation und in diesem Zusammenhang die Fragen, welche Textsorten die untersuchten Lehrwerke anbieten, ob und auf welche Art und Weise sie den Lerner für fachspezifische Textsortenmerkmale sensibilisieren, inwieweit sie fachliches Hintergrundwissen darbieten und interkulturelle Kompetenzen fördern und schließlich die Frage ihrer Einsetzbarkeit in der Fachübersetzerausbildung.
\end{abstract}

\section{Einleitung}

Der grenzüberschreitende Austausch von Waren und Dienstleistungen ist längst zu einer Selbstverständlichkeit geworden, und es wird immer deutlicher, dass die zunehmende Internationalisierung mit einem ansteigenden Bedarf an fachsprachlichem Können einhergeht. Dies gilt sowohl für die internationale Wirtschaftskommunikation, die inzwischen sehr differenzierte Kompetenzen voraussetzt, als auch für die Kommunikation im Bereich der Wissenschaft, Technik und Rechtsprechung.

Daraus entsteht für die Auslandsgermanistik zum einen ein verstärkter Bedarf an fachsprachlichem Unterricht, etwa in Form von DaF-Lehrveranstaltungen wie Wirtschaftsdeutsch, wissenschaftliches Schreiben, DSH-Training usw., zum anderen aber auch ein ständiger Lern- und Aktualisierungsbedarf im Bereich der Fachsprachenübersetzung.

Vieles deutet darauf hin, dass die Motivation zum Deutschlernen und Deutschstudium im Ausland nicht mehr primär philologisch begründet ist; vielmehr sind die Sprachlernmotive im instrumentellen Bereich zu suchen, etwa in der Perspektive 
des beruflichen und z.T. auch akademischen Vorankommens. Bereits in den 1980er Jahren haben interne Analysen des Goethe-Instituts eine große Nachfrage nach fachbezogenen Deutschkursen mit wirtschaftlicher Thematik ermittelt (vgl. Spillner 2005: 286 f.). Für die Zielgruppe der italienischen Studierenden, die in der vorliegenden Untersuchung im Mittelpunkt steht, ergab meine im Wintersemester 2007/08 an der Universität Bologna durchgeführte Spracheinstellungsuntersuchung, dass die Studierenden dem Deutschen einen hohen beruflichen Wert zuschreiben, und zwar sowohl bei der Angabe der Sprachlernmotive als auch bei spontanen Assoziationen ${ }^{1}$ (vgl. Rocco 2010). Diese Ergebnisse sprechen gleichfalls für einen verstärkten Bedarf an fachsprachlichen und besonders wirtschaftssprachlichen Lehrveranstaltungen. Dieser Bedarf wird im universitären Bereich zwar weitgehend wahrgenommen, doch die curricularen Konsequenzen stellen sich auch nach der Studienreform vielerorts eher im Schneckentempo ein. Bachelorkurse wie »Deutsch im Beruf« bzw. »Wirtschaftsdeutsch « und Masterstudiengänge zur Wirtschafts- bzw. Unternehmenskommunikation sind bei Weitem nicht flächendeckend und gehören eher in Ausnahmefällen zum Standardlehrangebot für Germanisten bzw. Deutschstudierende im Ausland. ${ }^{2}$

Ebenso groß ist der Bedarf an Wirtschaftsdeutsch im Bereich der Fachübersetzung. Sylvia Reinart berichtet, dass laut Technical Communicator's Forum für das Jahr 2000 ein Viertel der Übersetzungskosten der EU-Länder allein in Deutschland anfiel, und sie schreibt das der exportorientierten deutschen Wirtschaft zu:

»Ein Großteil der Übersetzungen sind Fachübersetzungen, wobei das Fach Wirtschaft neben Technik und Recht zu den am häufigsten genannten Arbeitsgebieten zählt.« (Reinart 2009: 243 f.)

Anliegen des vorliegenden Beitrags ist allerdings nicht die Frage, inwieweit sich der zunehmende Fachkommunikationsbedarf im konkreten Lehrangebot spiegelt, sondern vielmehr die konkrete Gestaltung des Fremdsprachenunterrichts im

${ }^{1}$ Bei der Frage »Was assoziieren Sie mit folgenden Sprachen«? wurde Deutsch (neben Japanisch) deutlich häufiger als andere zur Bewertung stehende europäische und außereuropäische Sprachen mit Effizienz, Wirtschaftskraft, Arbeit und mit wirtschaftlichem und technischem Fortschritt assoziiert. Auch in der repräsentativen Meinungsumfrage zu deutsch-italienischer Selbst- und Fremdwahrnehmung, die im Auftrag der Deutschen Botschaft Rom durchgeführt wurde, sind die auf diese Werte bezogenen Angaben stark vertreten (vgl. von Brandt 2011: 4).

2 Da dieser Beitrag von der auslandsgermanistischen Perspektive Italiens ausgeht, seien hier einige Beispiele für die curriculare Umsetzung des Bedarfs nach Fachsprachen und Unternehmenskommunikation an italienischen Universitäten angeführt: der von Federica Missaglia seit 2005/06 geleitete Masterstudiengang »Deutsch für die internationale Wirtschaftskommunikation« an der Università Cattolica del Sacro Cuore in Mailand und der Masterstudiengang »Unternehmenskommunikation« an der Universität Modena (vgl. Hornung 2007). 
Bereich Wirtschaftsdeutsch. Der Ausgangspunkt der Untersuchung, deren Ergebnisse im Folgenden vorgestellt werden sollen, war die Frage, wie fachspezifische Text- und Textsortenkompetenzen in den aktuellen Lehrwerken für Wirtschaftsdeutsch vermittelt werden. ${ }^{1}$ Die einzelnen Untersuchungsziele wurden ausgehend von den folgenden Fragen operationalisiert:

1. Welche Teilbereiche der schriftlichen Wirtschaftskommunikation stehen bei den untersuchten Lehrwerken im Vordergrund?

2. Welche Textsorten bieten die analysierten Lehrwerke an? Wie werden sie dem Lerner nähergebracht und welche Rolle spielt die Arbeit an der Textkompetenzvermittlung?

3. Inwiefern vermitteln die untersuchten Materialien auch interkulturelle Kompetenzen und fachliches Hintergrundwissen?

4. Eignen sich die analysierten Lehrwerke auch für den Einsatz im fachsprachlichen Übersetzungsunterricht?

Analysiert wurden die aktuellen Lehrwerke für Wirtschaftsdeutsch von drei Verlagen:

1. Dialog Beruf 3 vom Hueber-Verlag, Lernniveau B1/B2 (im Folgenden DB3),

2. Unternehmen Deutsch Aufbaukurs vom Klett-Verlag, Lernniveau B1/B2 (UDA),

3. Wirtschaftskommunikation Deutsch vom Langenscheidt-Verlag, Lernniveau $\mathrm{B} 2 / \mathrm{C} 1$ (WKD).

Der Auswahl der Lehrwerke wurden vier Kriterien zugrundegelegt:

- Als erste Voraussetzung wurde das Mindestzielniveau B2 festgelegt, da niedrigere Lernstufen die Arbeit mit authentischen Textsorten der Wirtschaft erheblich einschränken und die entsprechenden Zielkompetenzen nicht über die elementare Verständigung am Arbeitsplatz hinausgehen. ${ }^{2}$

1 »Wirtschaftsdeutsch« wird hier zunächst als Oberbegriff für unterschiedliche in Frage kommende Benennungen des Lerngegenstands in den berufsspezifischen Kursen wie etwa »Wirtschaftskommunikation Deutsch «, »Unternehmenskommunikation «, »Deutsch für den Beruf«, »Deutsche Geschäftssprache« benutzt. Auf den Begriff der Wirschafts(fach)sprache im Allgemeinen wird in Abschnitt 2 eingegangen.

2 Wie den kann-Beschreibungen des Gemeinsamen Europäischen Referenzrahmens für Sprachen im Bereich Beruf zu entnehmen ist, können die für das Zielniveau B1 angesetzten Kompetenzen eher als Vorstufe zu fachsprachlicher Kompetenz gesehen werden; so werden z. B. die Lese- und Schreibkompetenz wie folgt definiert: Lesen: »kann innerhalb des eigenen Arbeitsgebiets die allgemeine Bedeutung von nicht-alltäglichen Briefen und theoretischen Artikeln verstehen «; Schreiben: »kann in einer Besprechung oder einem Seminar ziemlich genaue Notizen machen, wenn die Thematik vertraut und vorhersehbar ist «. Auf dem B2-Niveau heißt es dagegen bei der Teilkompetenz Lesen: »kann die meisten Schriftwechsel, Berichte und Produktbeschreibungen, die er/sie erhält, verstehen «, und beim Schreiben: »kann alle Routineanfragen hinsichtlich Waren oder Dienstleistungen bewältigen« (vgl. GER). 
- Zweitens wurden von den bekannten deutschen DaF/DaZ-Verlagen jeweils nur die jüngsten Lehrwerke ausgewählt.

- Drittens schloss das Kriterium der Einsetzbarkeit als kurstragendes Lehrwerk die Lehrmittel aus, die nur Teilfertigkeiten trainieren (etwa Verhandlungsgeschick, Fachwortschatz, Geschäftskorrespondenz).

- Viertens beschränkte sich die Auswahl auf die Lehrwerke, die von 2008 bis 2010 in meinem Wirtschaftsdeutsch-Unterricht an der Universität Bologna erprobt werden konnten. Die Möglichkeit, bei den untersuchten Textsorten und Aufgabentypen konkrete Unterrichtserfahrung heranzuziehen, erwies sich bei der Analyse als ein bedeutender Vorteil.

Die Vorgehensweise sei im Folgenden kurz skizziert: Zunächst wurden beide Lehrwerke mit dem Zielniveau B2 im Hinblick auf die dargebotenen Textsorten, die entsprechenden Aufgabenstellungen und weitere oben genannte Fragen analysiert und gegenübergestellt. Das Hinzuziehen des dritten Lehrwerks mit dem höheren Zielniveau C1 ermöglichte, die jeweiligen Besonderheiten der untersuchten Lehrwerke auf einer breiteren Vergleichsbasis zu beleuchten. Im Mittelpunkt stand dabei, wie bereits erwähnt, die schriftliche Wirtschaftskommunikation. Die dargebotenen Formen der mündlichen Interaktion wurden bei der Analyse nicht berücksichtigt, da die gesprochene Sprache eine grundverschiedene Herangehensweise voraussetzt und die Auseinandersetzung mit Fragen erforderlich macht, die den Rahmen dieser Arbeit sprengen würden.

\section{Wirtschaftsfachsprache(n) vs. Wirtschaftsdeutsch als DaF-Unter- richtsfach}

Im Vorfeld zur Lehrwerkanalyse drängt sich die Frage auf, welche Aspekte der Wirtschaftsfachsprache(n) im Deutschunterricht für Nicht-Muttersprachler überhaupt vermittelt werden (sollten). Um diese Frage zu beantworten, wenden wir uns zunächst dem allgemeinen, nicht DaF/DaZ-spezifischen Begriff der Wirtschaftsfachsprache zu. In der Literatur besteht weitgehend Einigkeit darüber, dass es sich hierbei um ein sehr komplexes und wenig übersichtliches Feld handelt, dessen vorliegende Beschreibungen trotz relativ früher Anfänge manches Forschungsdesiderat offen lassen (vgl. z. B. Messings Ausführungen zur Wirtschaftslinguistik $(1932,1928)$ und Siebenscheins (1936) zur Wirtschaftsgermanistik).

In der Kommunikationswissenschaft und besonders in der Erforschung der interkulturellen Kommunikation sowie der Unternehmenskommunikation finden sich zwar wertvolle Anregungen, doch vom sprachwissenschaftlichen und besonders vom sprachvergleichenden Standpunkt her ist der Bereich der Wirtschaftstexte im Vergleich zu anderen fachsprachlichen Texten als weniger erforscht anzusehen (vgl. Reuter 2001: 578; Brünner 2000: 1; Rega 2008: 123; Bungarten 1997: 7; Koch 1997: 46). 
Die Gründe dafür liegen z. T. in der Vielfalt und Komplexität der möglichen »wirtschaftlichen « Handlungen und Interaktionen, die zahlreiche und im Hinblick auf den Fachlichkeits- sowie Standardisierungsgrad sehr unterschiedliche Textsorten hervorbringen bzw. hervorgebracht haben. Reuter formuliert es in seinem Handbuch-Beitrag zum Thema »Wirtschaftstexte« besonders prägnant:

»Wirtschaft ist überall und deshalb gibt es auch überall Texte, die auf die eine oder andere Weise mit ihr zu tun haben«. (Reuter 2001: 573)

Laut Brünner (2000: 1) ist die Wirtschaft »ein zentraler Bereich gesellschaftlichen Handelns « und, da das Wirtschaftsgeschehen alle Bereiche von Gesellschaft und Politik beeinflusst, auch »ein zentraler Ort gesellschaftlicher Kommunikation«. »Die wirtschaftliche Kommunikation«, so die Autorin, »dürfte einen hohen Prozentsatz dessen ausmachen, was überhaupt an Kommunikation in der Gesellschaft stattfindet $\ll$.

Spillner (2002) beschreibt die Schwierigkeit, den Objektbereich der Wirtschaftssprache abzugrenzen, wie folgt:

»Wenn man Kommunikationsbereiche von der Theorie des Wirtschaftsrechts über die Terminologie des Warenhandels, die Börsensprache und die Handelskorrespondenz bis hin zur wirtschaftlich-technischen Dokumentation und zur Produktwerbung einbezieht, ergibt sich in der Tat ein sehr heterogener Objektbereich. Hinzu kommt, daß einige wirtschaftssprachliche Textsorten stark journalistisch und wissensvermittelnd-popularisierend geprägt sind.« (Spillner 2001: $145 \mathrm{f}$;; vgl. auch Spillner 2005: 289; Kovtyk 2002: 108)

Die Heterogenität der Textsorten und Kommunikationsformen ist zwar kein exklusives Problem der Wirtschaftssprache, doch sie wird von einigen zusätzlichen Faktoren beeinflusst, die weder im Bereich der grundsätzlich konservativen Rechts- und Verwaltungstexte noch in dem der akademischen Textproduktion eine vergleichbare Rolle spielen: Der erste und wichtigste Faktor ist die rasante Dynamik des wirtschaftlichen Geschehens, die als Motor der Sprachentwicklung und -veränderung wirkt. Sie führt zu schnellem Wandel der Textsortenkonventionen und bringt immer neue Formen der schriftlichen und mündlichen Kommunikation hervor. Außerdem beschleunigt sie die Prozesse der Neologismenbildung und der Entlehnung von Fremdwörtern, besonders von Anglizismen, die selten eine schnelle Aufnahme in die Fachwörterbücher und Terminologiedatenbanken erfahren. Dies ist zum Teil sicher auf sprachpuristische Tendenzen zurückzuführen (vgl. Reinart 2009: 245), zum Teil aber auch der Vorsicht der Wörterbuchautoren zu danken, da sich unter Neubildungen und Lehnwörtern aus dem Wirtschaftsleben oft auch sog. Eintagsfliegen finden. Während z. B. im Bereich der Gesetzgebung und der institutionellen Kommunikation jeweilige normsetzende Instanzen für einen Mindestgrad an Einheitlichkeit sorgen, führt der Konkurrenzkampf der Unternehmen nicht selten zu gewollter Diversität - 
etwa im Sinne einer bewussten Arbeit am Image bzw. an der corporate identity eines Unternehmens (vgl. dazu Schürmann 1994), in deren Rahmen oft auch Bemühungen um einen eigenen, sofort erkennbaren und unverwechselbaren Sprachgebrauch beobachtet werden können.

Im Vergleich $\mathrm{zu}$ den anderen Fachsprachenbereichen ist noch eine weitere Besonderheit der Wirtschaftskommunikation zu erwähnen: Zwar kann die fachexterne Kommunikation vom Fachmann zum Laien durchaus auch Bestandteil der juristischen und akademischen Kommunikation sein; für die Wirtschaftskommunikation (und z. T. auch im Bereich der Gebrauchstechnik) ist diese Konstellation allerdings von essenzieller Bedeutung, da praktisch jeder an ihr teilnimmt, sei es als Endverbraucher, sei es als Arbeitnehmer. Nicht zuletzt aus diesem Grund sind in vielen Sparten der Unternehmenskommunikation die Übergänge zwischen fach- und allgemeinsprachlichen Texten fließend; so zeichnen sich z.B. selbstdarstellende Texte (z. B. im Bereich der Öffentlichkeitsarbeit und der Werbung) oft gerade durch einen sehr zugänglichen allgemeinsprachlichen Stil aus. Wie schlägt sich nun die beschriebene Vielfalt und Heterogenität der Wirtschaftsfachsprachen und -bereiche in der didaktischen Zielsetzung und Textauswahl nieder? Dass die Fachsprachen bzw. Fachtexte der Wirtschaft in all ihrer Komplexität nicht Gegenstand des Deutschunterrichts sein können, wurde bereits mehrfach festgestellt und geht auch aus den - wenn auch abstrakten - Zielbeschreibungen des GER hervor.

Reuter/Schröder/Tiittula beobachten in diesem Zusammenhang:

»Wirtschaftskommunikation stellt mit ihrer horizontalen Auffächerung (in Disziplinen und Fachbereiche) und mit ihrer vertikalen Schichtung (in verschiedene Verwendungssituationen, Fachstile und Register) sowie durch die Verflechtung mit anderen Fachsprachen ein sehr komplexes Feld dar, das nicht in seiner Gesamtheit Gegenstand im Fremdsprachenunterricht sein kann." (Reuter/Schröder/Tiittula 1991: 111; vgl. auch Frenser 1991: 236)

Eine praxisnahe Antwort auf die Frage, was genau in den aktuellen Lehrwerken für Wirtschaftsdeutsch vermittelt wird und wie dies geschieht, soll die folgende Darstellung der Untersuchungsergebnisse liefern.

\section{Lernziele und dargebotene Textsorten}

Als ihr Hauptziel definieren die untersuchten Lehrwerke praxisnahes Lernen und sprachliche Handlungsfähigkeit. So heißt es in DB3, dass dem Lehrwerk eine groß angelegte Analyse des Sprachbedarfs in deutschen Unternehmen zugrunde liege (Vorwort: 3). Als Schauplatz des Lehrwerks wurde ein reell existierendes Unternehmen, der Pumpenhersteller Allweiler AG aus Radolfzell am Bodensee, gewählt. Ein konkreter Geschäftsfall (ein Auftrag aus Japan) durchzieht als Hand- 
lungsfaden das Lehrwerk und bezieht bei den einzelnen Arbeitsetappen verschiedene Bereiche des Unternehmens ein (Auftragsabwicklung, Projektplanung, Produktion, Lieferung usw.). Auch in den beiden anderen Lehrwerken wird die sprachliche Handlungsfähigkeit i. d. R. an Fallbeispielen authentischer bzw. leicht adaptierter oder gekürzter betrieblicher Abläufe geübt. UDA definiert sich, gleichfalls im Einklang mit den Vorgaben des GER, als ein berufsorientiertes Lehrwerk, das »eine umfassende Handlungsfähigkeit am Arbeitsplatz « sowie »berufssprachliche und interkulturelle Kompetenz« vermittelt (vgl. Vorwort zum Lehrbuch) und schnelle Anwendung der erworbenen Fertigkeiten anstrebt, so dass Lernen und Anwenden eher parallel erfolgen sollen (vgl. Lehrerhandbuch: 5). Ähnlich kann man im einleitenden Kapitel zu WKD (»Zum Buch«: 4) lesen:

"Die Inhalte und Aufgaben von Wirtschaftskommunikation Deutsch orientieren sich an konkreten Handlungssituationen im Unternehmen und an den sprachlichen Anforderungen, die sich dort stellen.«

Die lange Danksagung auf Seite 3 ergeht an gut 40 Unternehmen und Einrichtungen (u.a. Großunternehmen wie BMW, SAP, Henkel, mehrere Messegesellschaften, die Deutsch-Niederländische Handelskammer Düsseldorf usw.), die für das Lehrwerk »Recherchen in ihrem Haus ermöglicht und/oder Materialien zur Verfügung gestellt haben « (2).

Bei einer ersten Durchsicht der Lehrwerke fällt auf, dass alle drei Verlage bemüht sind, die Realität der Unternehmenskommunikation möglichst treu nachzuzeichnen. Dies spiegelt sich zunächst in der Form der dargebotenen Texte wider: Auch Texte, die wegen ihrer Länge und/oder Komplexität adaptiert werden mussten, ${ }^{1}$ wirken authentisch. Die grafische Gestaltung ist im Vergleich zu den jeweils älteren Wirtschaftsdeutsch-Lehrwerken derselben Verlage (z. B. Dialog Deutsch für den Beruf/Hueber), aber auch zu den aktuellen Lehrbüchern für Wirtschaftsfranzösisch und für Wirtschaftsitalienisch, ${ }^{2}$ bedeutend realitätsnäher.

Ein weiteres, sehr ausgeprägtes Merkmal der drei Lehrwerke ist die Textsortenvielfalt. Es sind sowohl Texte der externen Unternehmenskommunikation vertreten als auch Beispiele für die interne Unternehmenskommunikation auf verschiedenen Betriebsebenen und unterschiedlichen Formalitätsgrades.

Im Lehrwerk DB3 umfasst die externe Unternehmenskommunikation die Kontakte des Schauplatz-Unternehmens Allweiler AG zu seiner Umwelt im weiteren Sinne, d.h. zu einem breiten, nicht genauer definierten Adressatenkreis potenti-

1 Ein Beispiel hierfür ist der in seinem Umfang reduzierte unternehmensinterne Beurteilungsbogen in UDA (100f.). Im Lehrerhandbuch (44) wird die Kürzung damit begründet, dass Beurteilungsbögen normalerweise mehrere Seiten umfassen, die Beurteilungsskalen in der Realität länger und die Beurteilungsmerkmale weiter aufgefächert sind.

2 Z. B. Affaires.com/CLE international (2003, Niveau B1), Affaires à suivre/Hachette (2001, B1/B2), L'italiano in azienda/Guerra (2002, B1/B2). 
eller Kunden oder Lieferanten, zu anderen Unternehmen als Geschäftspartnern sowie zu Ämtern, Behörden und Banken. Dazu gehören folgende Textsorten: Unternehmenspräsentation, Geschäftsbericht, Produktbeschreibung, Prospekt, die grundlegenden Geschäftsbriefsorten (Anfrage, Auftrag, Reklamation usw.), Begleitschreiben der Auftragslogistik und Lieferung (Versandanzeige, Versandpapiere), Tagungsprogramm, Texte aus dem Bereich des Zahlungsverkehrs (Akkreditiv) und der Messekommunikation (Hallenübersicht, Formular zur Standanmeldung, Messebeschreibung, Messebericht), Bewerbungsunterlagen (Lebenslauf, Anschreiben) und die Erläuterungen zur Lieferklausel FOB (frei an Bord). Der relativ große Anteil an unternehmensinternen Formen schriftlicher Kommunikation korrespondiert mit der Zielgruppenbestimmung - in der Internetpräsentation des Lehrwerks wird das Zielpublikum wie folgt beschrieben:

"Lerner, die sich auf eine zeitlich begrenzte oder auf eine dauernde Berufstätigkeit in deutschsprachigen Ländern vorbereiten oder Deutsch als berufliche Arbeitssprache in ihrem Heimatland einsetzen möchten.« (www.hueber.de)

Die meisten Texte umfassen schriftsprachliche Handlungen aus dem unternehmensinternen Arbeitsalltag, so z. B. unternehmensinterne Rundschreiben, halbformelle Zettel an Kollegen bzw. Mitarbeiter, ein intern vereinbartes Praktikumsprogramm, eine Checkliste zur Messevorbereitung, und außerdem hoch standardisierte Textsorten wie Leistungsbeurteilung, Antrag auf Mehrarbeit, Betriebsanweisung zur Arbeitssicherheit sowie auch solche, die arbeitsrechtliche und verwaltungstechnische Fragen regeln, z. B. Arbeitsvertrag, Kündigung und das Formular »Anhörung des Betriebsrats vor einer Einstellung «. Texte im Stil von Zeitungsberichten und -interviews kommen in DB3 lediglich im Rahmen des Prüfungstrainings vor (d.h. in den mit »Training Zertifikat Deutsch für den Beruf « betitelten Doppelseiten am Kapitelende).

$\mathrm{Zu}$ den ebenfalls vielfältigen Textsorten im Lehrwerk UDA gehören neben den gerade angeführten Beispielen für unternehmensexterne Kommunikation auch folgende Textsortenbeispiele: Begrüßungsbrief, Versandauftrag, gesetzliche Gewährleistung, Liefer- und Geschäftsbedingungen, Leasingvertrag als Beispiel einer hoch spezialisierten Textsorte, Arbeits- und Zwischenzeugnis und als Hintergrundinformation ein Text mit dem Titel »Geheimcodes der Arbeitszeugnisse«. Dazu kommen auch mehrere Texte aus den Bereichen des Zahlungsverkehrs und der Finanzverwaltung (Überweisungsauftrag, Einzugsermächtigung, Gehaltsabrechnung), Messestatistiken und -grafiken usw. Stellvertretend für die interne Unternehmenskommunikation finden sich im Lehrwerk neben den auch in DB3 vertretenen Notizzetteln (z. B. Messenotiz) weitere konkrete Beispiele aus dem Berufsalltag, wie z. B. Sitzungsprotokoll, tabellarische Auftragsübersicht in Form von Excel-Tabelle, Personaleinsatz-Tabelle, Gesprächsnotiz, Merkzettel, Organigramm, Beurteilungsbogen, Bedienungsanleitung und Präsentations- 
slides. Hinzu kommen die sogenannten Journalseiten am Ende jedes der insgesamt zehn Kapitel, die jeweils zwei Beiträge in Form von Zeitungs- und Reiseführertexten, Unternehmenspräsentationen, Lexikoneinträgen und Ratgebertexten enthalten (z. B. der oben genannte Text zu den Textkonventionen der Arbeitszeugnisse). Während DB3 und UDA sich auch als Vorbereitungswerke für die B2-Prüfung »Zertifikat Deutsch für den Beruf « verstehen, zielt das Lehrwerk Wirtschaftskommunikation Deutsch (WKD) auf die »Prüfung Wirtschaftsdeutsch" mit dem Kompetenzniveau $\mathrm{C} 1 \mathrm{ab}$, die im Bereich der schriftlichen Wirtschaftskommunikation u. a. eine umfassende und differenzierte Leseverstehenskompetenz voraussetzt. ${ }^{1}$ Den hohen Prüfungsanforderungen entspricht bei WKD ein höherer Fachlichkeitsgrad der Texte, grafischen Darstellungen und auch Hintergrundinformationen. WKD bietet zum einen bereits oben erwähnte Textsorten der externen und internen Unternehmenskommunikation wie Standardformen des Geschäftsbriefs, Produktbeschreibung, Unternehmensporträt und Gesprächsprotokoll, die hier allerdings in mehreren Varianten vorkommen und einen höheren Schwierigkeitsgrad haben. Zum anderen beinhaltet es Textsorten, die in beiden B2-Lehrwerken nicht auftreten und fachfremden Lernern weniger geläufig sein dürften: Briefe wie z. B. die Bitte um wirtschaftliche Auskunft bei der Handelskammer, Aufnahme der Nachmessekontakte, Kontaktaufnahme-Schreiben an potentielle Geschäftspartner und Vorstellung des neuen Produkts bei Stammkunden. Darüber hinaus kommen auch Texte vor, die an Fachlichkeit weit über die übliche Geschäftskorrespondenz hinausgehen, so z. B.: Gewinn- und Verlustrechnung eines Unternehmens für ein Geschäftsjahr in tabellarischer Form, Bank-Auskunft über ein Unternehmen, Marketingkonzept-Präsentation, Produktbriefing, Fragebogen zur Bewertung der Kundenattraktivität und ferner auch komplexe Grafiken, die betriebswirtschaftliche Kenntnisse mitvermitteln, wie etwa das mehrschichtige Kreisdiagramm »Die Facetten der Markterschließung « (187), das Kurvendiagramm »Produktlebenszyklus« (157) und die Portfolio-Matrix der Kundenprofile (204).

Das breite Spektrum von Themen und Textsorten, das alle drei Lehrwerke und insbesondere UDA und WKD kennzeichnet, bietet dem Kursleiter die Möglichkeit, die Textauswahl optimal an die jeweilige Zielgruppe anzupassen. Sie birgt

1 Der Leseverstehensteil der Prüfung besteht aus mehreren Textsorten, die jeweils selektives, detailliertes und globales Lesen trainieren, wie etwa Pressetexte bzw. -mitteilungen (Firmenportraits, Texte über Produkte, Märkte, Geschäftsergebnisse, Trends \& Themen), kundenorientierte Texte wie Prospekt und Werbematerial und betriebsinterne Unternehmenstexte wie z. B. Protokoll, Notiz und Memo. Die Schreibaufgabe testet die Fertigkeit, ein Kontaktschreiben, eine Anfrage, eine Pressemitteilung o. ä. nach Vorgaben zu verfassen (vgl. http://www.dihk-bil-dungs-gmbh.de/ index.php?id=8). 
jedoch auch Tücken, und zwar nicht nur für leistungsschwächere Lerner und Autodidakten, die die Fülle des Materials vermutlich eher schwer überblicken, sondern auch für Lehrkräfte mit wenig Erfahrung im Fach Wirtschaftsdeutsch.

\section{Textkompetenz, interkulturelle Kompetenz und fachliches Hinter- grundwissen als Lernziele}

Weder DB3 noch die beiden anderen Lehrwerke bezeichnen die Textkompetenz als primäres Lernziel. Im Lehrerhandbuch zu DB3 wird im Abschnitt »Das Prinzip des lebensnahen Unterrichts«, Unterabschnitt »Lebensnahe Textarbeit« (15f.), von der instrumentalen Funktion der Texte ausgegangen:

»Er [der Text] kann Anlass, ja sogar Modell für die schriftliche und mündliche Produktion [...] sein. Aber er ist kein Lernziel. [...] Deshalb sind Texte in der Regel erledigt, wenn die dazugehörigen Aufgabenstellungen erledigt sind.«(16)

Weiter heißt es dann etwas überspitzt, dass Textanalysen, weiterführende Worterklärungen usw. dem Prinzip des lebensnahen Unterrichts widersprechen. Diese Programmatik, die der Textanalyse den Praxisbezug abzusprechen und die (rezeptive) Fachtextkompetenz nicht zu den Lernzielen zu zählen scheint, wird allerdings bereits bei der Darstellung der Arbeitsbuchziele abgeschwächt, ${ }^{1}$ und bei der Untersuchung der Lehrwerksaufgaben erweist sich dann, dass die Aufgaben, die für spezifische Vertextungsmuster sensibilisieren, doch sehr wohl und in vielerlei Hinsicht auch zu sprachlicher Handlungsfähigkeit beitragen. ${ }^{2}$ In der folgenden Übersicht liegt der Schwerpunkt auf den Aufgaben, die - mehr oder weniger direkt - die Ausbildung textspezifischer Sprachkompetenz fördern. Ein gutes Beispiel für didaktische Progression liefert die erste Begegnung mit der Textsorte Geschäftsbrief in DB3: Auf Seite 12 sieht man drei aus Briefkopf und ersten Textzeilen bestehende Briefausschnitte - eine Anfrage, eine Nachricht über die Lieferungsverzögerung und eine Versandanzeige -, die einen ersten Einblick in die Makrostruktur und textspezifische Anredekonventionen gewähren. Die entsprechende Aufgabe besteht in der Zuordnung der Briefe zu den jeweils zuständigen Mitarbeitern verschiedener Abteilungen (Auftragsabwicklung, Einkauf, Versand) und führt durch die Thematisierung der potentiellen Briefadressaten progressiv in die Problematik der Textsorten und -funktionen ein. Bei einer weiteren Aufgabe, die gleichzeitig für Textsortenmerkmale und Textfunktion

1 Im Unterabschnitt »Lebensnahe Übungsansätze« (16) ist zu lesen: »Die vorwiegenden Intentionen der Arbeitsbuchübungen sind die grammatische Regelfestigung, die Einübung des Wortschatzes und - in geringerem Umfang - die (Lese-) Textarbeit.«

2 Zur Rolle der Textkompetenz und der ungenügend beachteten Bedeutung der Textarbeit im Fremdsprachenunterricht vgl. Fandrych/Thurmair 2010: 350-352. 
sensibilisiert (20), sollen 12 Textausrisse ${ }^{1}$ aufgrund ihrer charakteristischen Merkmale als betriebliche Textsorten (Reklamation, Anfrage, Rechnung, Bericht, Rundschreiben, Bewerbung, Einladung, Hotelprospekt...) identifiziert werden. Die Fragen lauten, um welche Art von Eingangspost es sich handelt und woran man diese Textsorten auf einen Blick erkennen kann, wobei zu den Lösungsmöglichkeiten sowohl sprachliche und inhaltliche als auch makrostrukturelle und grafische Hinweise zählen (z. B. Zahlen und tabellarische Gestaltung $\rightarrow$ Rechnung, »nicht zu unserer Zufriedenheit $\leadsto \rightarrow$ Reklamation). Die Aufgaben zu dem auf Seite 61 abgedruckten Akkreditiv-Faksimile thematisieren ebenfalls nicht nur inhaltliche, sondern auch strukturbezogene Textmerkmale (z. B. »In welcher Zeile steht, 1) bis wann die Deutsche Bank Osaka die Forderungen von Allweiler AG garantiert, 2) welchen Höchstbetrag sie garantiert, 3) worauf Allweiler AG besonders achten soll« usw.). Wenig Berücksichtigung finden die Textstrukturmerkmale dagegen in der Aufgabenstellung zum Bewerbungsschreiben (17), die hauptsächlich auf die inhaltlichen Bausteine einer Bewerbung eingeht: Der Lerner soll herausfinden, welche der im beiliegenden Lebenslauf vorhandenen Informationen auch im Bewerbungsschreiben erwähnt werden, wie der Bewerber seine Bewerbung begründet, was ihn interessiert, was er von dem Praktikum, um das er sich bewirbt, erwartet usw.

Diese Kurzdarstellung soll durch ein weiteres Aufgabenbeispiel ergänzt werden, das auch sozio- und pragmalinguistische Kompetenz im Sinne eines situationsangemessenen Sprachgebrauchs fördert: Im Fax auf Seite 59 kontaktiert eine Allweiler-Mitarbeiterin einen ihr offensichtlich gut bekannten Ansprechpartner des Partnerunternehmens und stellt Nachforschungen wegen eines nicht eingegangenen Akkreditivs an. Zu diesem halbformellen Schreiben bietet das Arbeitsbuch (58) die formelle Variante, und die Aufgabe besteht in der Zuordnung halbformeller und formeller Formulierungen mit derselben Mitteilungsabsicht.

Das Lehrwerk UDA definiert produktive und nicht rezeptive Fertigkeiten als vorrangige Lernziele. Im Lehrerhandbuch (6) wird betont, dass den Fertigkeiten Sprechen und Schreiben besonderes Gewicht beigemessen wird und von den rezeptiven Fertigkeiten der Schwerpunkt auf dem Hören liegt. Außerdem wird davon abgeraten, die Texte ausführlicher zu bearbeiten, als in der Aufgabenstellung vorgesehen ist. ${ }^{2}$

1 Beispiele für Textausrisse: »Bitte senden Sie uns ausführliche Produktinformationen. Für Ihre...« und »...die von Ihnen gelieferte BÜROLA x 23 nicht zu unserer Zufriedenheit arbeitet $\ll$.

2 So heißt es im Zusammenhang mit dem Text über die europäischen Krankenversicherungssysteme: »Es ist wichtig, dass KT [Kursteilnehmer] zuerst die Aufgabenstellung zur Kenntnis nehmen. KL [Kursleiter] achtet darauf, dass KT die Texte nicht durchlesen, sondern nach den geforderten Aufgaben durchsuchen.« (27) 
UDA zeichnet sich ebenfalls durch praxisorientierte Textarbeit aus; so soll der Lerner beispielsweise bei Stellenanzeigen, Allgemeinen Geschäftsbedingungen, Unternehmensporträts usw. praxis- bzw. handlungsrelevante inhaltliche Aspekte herausfiltern. Die Arbeit am Fachwortschatz erfolgt meistens im Rahmen des selektiven Lesens; z. B. in den drei kurzen Unternehmensporträts auf Seite 29 sollen die Unternehmenseckdaten markiert und in die mit "Unternehmen «, »Branche«, »Produkte «, »Märkte « beschrifteten Tabellenspalten eingetragen werden. Dieselbe Aufgabenstellung findet man auch bei dem Text zu den Rechtsformen eines Unternehmens, wobei die Ausdrücke im Tabellenkopf allerdings einen höheren Fachlichkeitsgrad aufweisen: »Grundkapital«, »Organe«, »typische Unternehmensgröße «.

Ein Beispiel für gezielte Arbeit an textsortentypischen Strukturbausteinen bietet die Bearbeitung der Textsorte Vertrag. So heißt es beim »Service-Auftrag Berufskleidung « auf Seite 80: „Was haben Profitext und AWA zu folgenden Punkten vereinbart? 1) Vertragsgegenstand, 2) Liefermenge, 3) Artikel (...), 8) Zahlungsweise«, und beim Leasingvertrag auf Seite 88 lautet die erste Frage: »Wer least was von wem $\ll$ ?

Auch in UDA (114) soll der Lerner verschiedene Textausrisse mit den entsprechenden Textsorten verbinden (Anfrage, Angebot, Begleitschreiben, Bestellung, Bitte um Informationen, Lieferschein, Rechnung) und darüber hinaus auch den üblichen chronologischen Geschäftsablauf rekonstruieren - ein weiteres Beispiel dafür, wie sich die Arbeit an Textfunktionen und Textsorten mit handlungsorientiertem Fachunterricht verbinden lässt.

Als exemplarisch können die vielseitigen Aufgaben zum Bewerbungsschreiben (162 f.) bezeichnet werden, da sie den Erwerb dieser für viele Berufsprofile und -kontexte wichtigen Textsortenkompetenz ermöglichen. Der Lerner soll systematisch alle Elemente eines formellen Schreibens wie Absender, Betreff, Anrede, Einleitung, Schlusssatz und Grußformel und außerdem auch grundlegende inhaltliche Elemente (z. B. Motivation, eigene Erwartungen) suchen, wie in DB3 einen Vergleich mit den Angaben im Lebenslauf (160) anstellen und schließlich untersuchen, zu welchen Punkten der Stellenanzeige der Brief nichts aussagt und ob das Fehlen der betreffenden Informationen ein Problem darstellt.

Was in beiden Lehrwerken bei diesem Thema auch noch wünschenswert wäre, ist zum einen der Hinweis auf weglassbare und wegzulassende Personalien im Lebenslauf, da hinsichtlich der Angaben wie Beruf der Eltern, Religion, Familienstand usw. große interkulturelle Unterschiede bestehen. Ein weiteres Desiderat, das ich ebenfalls aus der Unterrichtspraxis ableite, wäre eine zielgerichtete Sensibilisierung für die formale Gestaltung der Bewerbungsunterlagen. Die Analysen der am PC erstellten Bewerbungsunterlagen der Studierenden haben ergeben, dass die formalen Textgestaltungsnormen von vielen entweder überhaupt nicht wahrgenommen oder als unbedeutend eingestuft werden, so dass die 
Präsentabilität der Bewerbungsbriefe und Lebensläufe von »Schönheitsfehlern« beeinträchtigt wird, die in der Praxis gerade bei diesen Textsorten streng sanktioniert werden. Um nur einige Beispiele hierfür zu nennen: unterschiedliche und den Textgestaltungskonventionen wenig angemessene Schriftarten, Schriftgrößen und Zeilenabstände, uneinheitliche Gliederungs- und Nummerierungssignale sowie Interpunktionszeichen bei Aufzählungen, uneinheitliche Groß- oder Kleinschreibung am Zellenanfang und Verschiebungen des Zelleninhalts bei tabellarischem Lebenslauf, unausgeglichene Textverteilung und z. T. auch Lichtbilder, die im gegebenen Kontext unangemessen wirken.

Im Unterschied zu beiden B2-Lehrwerken bietet WKD, wie bereits in Abschnitt 3 angedeutet, auch Texte und grafische Darstellungen an, die fachunerfahrenen Lernern wenig geläufig sein dürften. Im Hinblick auf die Textarbeit kann man als die größten Vorzüge dieses Lehrwerks abwechslungsreiche, praxisbezogene und oft fachlich anspruchsvolle Aufgaben bezeichnen, die explorative Herangehensweise und analytisches Denken fördern und transferfähige Aktivitäten einleiten. Damit gelingt es diesem Lehrwerk, das Prinzip des forschenden Lernens im vollen Umfang umzusetzen. Um dies an zwei Beispielen zu veranschaulichen: Die Doppelseite 36-37 zeigt das Konzernporträt der BMW Group, ergänzt von acht relativ komplexen Graphiken zum Geschäftsjahr 2005, die wichtige Unternehmensdaten wie Umsatz, Investitionen usw. veranschaulichen. Die darauf folgende Doppelseite präsentiert dieselbe Art von Informationen, diesmal allerdings die Unternehmensdaten zum Jahr 2006, in einer anderen Form, und zwar als gekürzten Geschäftsbericht. Der Lerner soll die unternehmensrelevanten Aufgaben für das Jahr 2006 aus dem Geschäftsbericht und die Vergleichsdaten für das Jahr 2005 aus den Grafiken herausfiltern und beide Gruppen von Kennzahlen einem analytischen Vergleich unterziehen und auf der Vergleichsgrundlage Hypothesen über positive und negative Entwicklungen des Unternehmens anstellen. Auch die Doppelseite 100-101 zielt auf analytische Gegenüberstellung zweier Informationsquellen ab: einerseits die Unternehmensgeschichte der Firma Kolb von 1932 bis heute, die u. a. auch konjunkturbedingte Schwankungen der Auftragslage beschreibt, und andererseits das Balkendiagramm »Lebenslauf der Konjunktur « zur wirtschaftlichen Entwicklung Deutschlands im Zeitraum 19492005 einschließlich von Hinweisen auf Ölkrisen, Rezessionszeiten und »Vereinigungsboom «.

$\mathrm{Zu}$ den Texten in WKD kann im Allgemeinen festgehalten werden, dass sie eine bedeutend höhere Fachwortdichte aufweisen. So erscheinen z. B. neben den in die Allgemeinsprache eingegangenen Ausdrücken (etwa »Umsatz«, »Absatz«, »Wachstum«), die auch in den Unternehmenspräsentationen in DB3 und UDA auftreten, auch Fachausdrücke aus Finanzen und Marketing (»währungsbereinigt«, »Umtauschanleihe«, »Premiumsegmente«). Ein weiterer Unterschied besteht in der Tatsache, dass beide B2-Lehrwerke Textrezeptions- und Textproduk- 
tionsphasen im Sinne vorlagenorientierter Textproduktion stärker verbinden (z. B. Schritt 1: Textbeispiel für Auftrag, Schritt 2: rezeptive Aufgaben, Schritt 3: Textproduktionsaufgabe, bei der ausgehend von bestimmten Vorgaben ein neuer Auftrag geschrieben werden soll). WKD scheint dagegen von einem höheren sprachlichen Umsetzungsvermögen und einem Basiswissen über geschäftsspezifische Textsorten auszugehen, so dass auf die skizzierte Form des imitierenden Lernens meist verzichtet wird. So bietet das Buch z. B. bei den beiden ersten Textproduktionsaufgaben (Kontaktaufnahme-Brief an potentielle Kunden und Schreiben an die Handelskammer zwecks Unterstützung bei der Suche nach Geschäftspartnern, 45) reichlich Redemittel und Makrostrukturhinweise, ohne jedoch entsprechende Muster- bzw. Beispielschreiben voranzustellen.

Stellenweise scheint die Text- und Aufgabenkonzeption des Lehrwerks allerdings eine höhere Fachkompetenz nicht nur anzustreben, sondern auch vorauszusetzen und dabei von einer - gemessen am vorgegebenen Eingangsniveau - zu hohen Sprachkompetenz auszugehen. So gestalten sich z. B. nach dem TeilnehmerFeedback in meinen Kursen die Erklärung zum »pull-through «-Marketing (»alle Stufen der Wertschöpfung vom Handel- und Weiterverarbeiter bis zum Endverbraucher werden in die Vermarktungskonzepte einbezogen«, 206) sowie die gesamte Präsentation der Marketingkonzept-Unterlagen des Unternehmens 3M auf Seite $206 \mathrm{f}$. als sehr schwierig. Dasselbe gilt für die Aufgabe, eine fachterminologisch dichte Bankauskunft über die finanzielle Situation eines Unternehmens auf Seite 139 auszuwerten, sowie für andere, fachlich wie sprachlich sehr anspruchsvolle Aktivitäten des Kapitels 12.

Zudem bietet WKD keine Grammatikübersicht und keine gezielte Grammatikoder Wortschatzarbeit, abgesehen von den häppchenweise in Kästchen angebotenen Rede- und Strukturmitteln (etwa: »auf...Wert legen«, »bereit sein zu...«). Diese haben zwar den unbestreitbaren Vorteil, sich nach dem Prinzip »Weniger ist mehr « und vor allem durch unmittelbaren Einsatz in der mündlichen oder schriftlichen Textproduktion sehr gut einzuprägen, reichen jedoch für Lerner ohne gute sprachstrukturelle Basis nicht aus.

Im folgenden Abschnitt (5) soll gezeigt werden, dass die beschriebenen Aspekte nicht in allen Lernkontexten als nachteilig anzusehen sind. Vielmehr führt die Analyse hier zu folgender Schlussfolgerung: Um die mannigfaltigen Ressourcen, die WKD bietet, optimal nutzen zu können, empfiehlt es sich, dass die jeweilige Zielgruppe möglichst fachliche bzw. fachkontextspezifische Vorkenntnisse mitbringt und vorzugsweise ein abgeschlossenes B2-Niveau vorweisen kann. Die obere Kompetenzgrenze kann dagegen durchlässiger gehandelt werden, da das Lehrwerk sprachlich und fachlich auch für Lerner mit einem guten C1-Niveau noch viel Lernstoff bietet.

Auch die beiden anderen Lehrwerke bieten fachspezifisches Hintergrundwissen. DB3 präsentiert sehr verschiedene Formen betrieblichen Schriftwechsels und 
ermöglicht hiermit auch das Kennenlernen wichtiger betrieblicher Normen und Abläufe, die ansonsten unternehmensexternen Personen kaum zugänglich sind (Antrag auf Mehrarbeit, Formular »Anhörung des Betriebsrats vor einer Einstellung « usw. - vgl. Abschnitt 2). Die erwähnten Journalseiten des UDA, aber z. T. auch andere Seiten, informieren z. B. über Rechtsformen für Unternehmen, die Bildungssysteme Deutschlands, Österreichs und der Schweiz, Steuern- und Sozialabgaben im europäischen Vergleich, die europäischen Umweltzeichen, die LKW-Maut in Europa, Vor- und Nachteile des Leasing, den Unterschied zwischen Gewährleistung und Garantie usw. und bieten außerdem aktuelle Bewerbungstipps. Besonders begrüßenswert im Sinne der Lernerautonomie-Förderung ist der Hinweis auf die Seite EURES - das europäische Portal zur beruflichen Mobilität (166) und die mehrsprachige Vorlage »Europass-Lebenslauf« (160), die auf eine 2004 gegründete Initiative der Europäischen Kommission mit dem Namen Europass zurückgeht und auch weitere beruflich wichtige Dokumente und Erläuterungen bietet (vgl. Lehrerhandbuch: 66). Der Lerner wird hier konstruktivistisch zum Selbststudium und zu eigenständiger Suche nach sprachlichen und fachlichen Informationen angeregt.

Abschließend sei noch auf die Förderung interkultureller Kompetenz eingegangen, sofern sie bei der Textarbeit berücksichtigt ist. Um zuerst einige Beispiele für interkulturell relevante Aufgabenstellung zu nennen: Einer der Hauptprotagonisten in DB3 ist Alfred Sekakane aus Transvaal, der sich in der ersten Lektion um ein Praktikum bewirbt und ins Praktikumsprogramm eingewiesen wird. In der letzten Lektion schreibt er einen Abschlussbericht und soll bei einer vom Betrieb organisierten Abschiedsparty ein Abschiedsgeschenk überreicht bekommen. Die Fragen zum Rundschreiben an alle Kollegen, in dem die Abschiedsfeier angekündigt und um Spenden zwischen 2 und 5 Euro für ein Abschiedsgeschenk gebeten wird, gehen explizit auf mögliche interkulturelle Unterschiede ein (123): »Würde es bei Ihnen in einem solchen Fall eine Verabschiedung geben? Welchen Charakter hätte sie? Würde es ein Geschenk geben? Wer würde es bezahlen? Was würden Sie schenken? Wäre auch bei Ihnen eine kleine Ansprache üblich«? UDA behandelt interkulturelle Aspekte meist implizit, stellenweise auch explizit ${ }^{1}$ (vgl. Lehrerbuch: 5). In WKD begegnet man zielgerichteter, expliziter Reflexion über interkulturelle Unterschiede bereits am Anfang des erstens Kapitels (15), und zwar im Text über kulturspezifische Gesprächsthemen und Tabubereiche bei der deutschchinesischen Kommunikation, der aus Müllers Band Interkulturelle Kommunikation (1991: 305 f.) stammt. Dem thematischen Einstieg dienen zehn Fragen zu beruflichen und privaten Aspekten des Lebens (berufliche Tätigkeit, Einkommenshöhe,

1 Z. B. in einem Tipp-Kästchen am Seitenrand kann man lesen: »In Deutschland findet man die Frage nach dem persönlichen Einkommen normalerweise indiskret. [...]« (103) 
Gesundheitszustand, Alter, Religion...), die privater, beruflicher und formeller beruflicher Vorstellung zugeordnet und auf ihre Angemessenheit bzw. Unangemessenheit in diesen Situationen hin überprüft werden sollen.

Um die Analyseergebnisse im Hinblick auf interkulturelles Lernen zusammenzufassen: Die drei Lehrwerke enthalten zwar interkulturell relevante Materialien und Aufgaben, von einer durchgehenden und systematischen Behandlung interkultureller Thematik kann jedoch nicht gesprochen werden. Die Unterrichtserfahrung in den Wirtschaftsdeutsch-Kursen an der Universität Bologna und insbesondere die Reaktionen der Studierenden auf einzelne Texte und Aufgaben haben zu der Annahme geführt, dass eine verstärkte Sensibilisierung für kulturell geprägte Kommunikationsformen die interkulturelle (Text-)Arbeit sinnvoll ergänzen könnte. Dies könnte durch die Auseinandersetzung mit den Fragen erfolgen, ob eine bestimmte sprachliche Kommunikationshandlung im Heimatland der Lerner üblich oder angebracht wäre, ob sie schriftlich oder mündlich erfolgen würde, in welcher Form usw. Entsprechende Fragen bzw. Aufgaben könnten auch explizit im Zusammenhang mit den Textsorten auftreten, bei denen bedeutende interkulturelle Unterschiede anzunehmen sind (z. B. Zwischenzeugnis, Gesprächsprotokoll, Praktikumsplan, unverbindliche Produktanfrage). Diese Aufgabentypen sind zwar keinesfalls abwesend, ${ }^{1}$ kommen jedoch insgesamt zu selten vor.

\section{Einsatzpotential in der Fachübersetzerausbildung}

In den vorigen Abschnitten wurde gezeigt, dass WKD eine Fülle authentischer Materialien bietet, die von den geläufigen Geschäftsbriefen und Gebrauchstexten bis hin zu Fachtexten im engeren Sinne reichen. Es ist zwar anzunehmen, dass der Lernstoffumfang das vorgesehene Unterrichtspensum vielerorts übersteigt und autodidaktisches Lernen ohne einen Kursleiter, der je nach Zielgruppe andere Schwerpunkte setzt, auch erschweren kann. Andererseits spricht vieles dafür, dass diese zurzeit auf dem DaF-Markt einzigartige Materialsammlung in fachsprachlichen Kursen für Übersetzer fruchtbar genutzt werden kann - und das gilt besonders und gerade für die oben erwähnten Texte bzw. Aufgaben, die sich für die beobachtete Zielgruppe als zu anspruchsvoll erwiesen.

Der Einsatz von DB3 und UDA in der Fachübersetzerausbildung kann ebenfalls empfohlen werden, insbesondere bei Lernergruppen philologischer Grundausrichtung, die mit der deutschen Unternehmensrealität noch nie in Berührung

1 Z. B. im UDA lautet beim Thema Arbeitszeugnis eine der Fragen: »Haben Sie schon ein Arbeitszeugnis bekommen «? (92), und in DB3 heißt es bei der schriftlichen Terminabsage: »Wie würde man in Ihrem Land einen vereinbarten Termin absagen bzw. verschieben «? (22) 
gekommen sind. Beide Lehrwerke bieten eine für die Unternehmenskommunikation in Deutschland repräsentative Auswahl an aktuellen und authentischen Materialien an und reduzieren zugleich die Fülle in Frage kommender schriftlicher Kommunikationsformen auf das Wesentliche.

Um das Potential der analysierten drei Lehrwerke in den wichtigsten Punkten zusammenzufassen: Erstens liefert die Gesamtheit der dargebotenen schriftlichen Kommunikationsformen einen ersten Überblick über die Textsorten, die im Wirtschaftsleben auftreten können - einschließlich derjenigen, die in der LernerMuttersprache (noch) nicht existieren oder eine geringere Vorkommenshäufigkeit aufweisen. ${ }^{1}$ Zweitens enthalten diese Lehrwerke vielfältige und größtenteils konstruktivistisch angelegte Aufgaben, die die fachsprachliche Kompetenz erhöhen können. Drittens bieten sie einen ersten Einblick in das nötige Hintergrundwissen, und viertens kann die stark ausgeprägte Realitätsnähe der grafischen Textgestaltung zur Erhöhung der Layoutkompetenz beitragen, die in der Übersetzungspraxis zunehmend an Bedeutung gewinnt (vgl. Kautz 2002: 18).

$\mathrm{Zu}$ den Einsatzmöglichkeiten im Unterricht können an dieser Stelle nur einige allgemeine Anregungen formuliert werden, da konkrete unterrichtsbezogene Entscheidungen nur mit Blick auf die jeweilige Zielgruppe getroffen werden können. Die dargebotene Materialsammlung kann den Kursleiter bei der Auswahl der Textsorten, die im Kurs behandelt werden sollen, unterstützen. Im Rahmen der Textsortenarbeit können die Kursteilnehmer gemeinsam diskutieren, welche Text(sorten)kompetenzen auf dem jeweiligen Übersetzungsmarkt besonders gefragt sind. Außerdem bieten sich die drei Lehrwerke als Ausgangsbasis für die eigenständige Suche nach Paralleltexten und für den Vergleich der jeweils spezifischen Vertextungsmuster an. So kann z. B. das in allen Lehrwerken behandelte Thema »Messen « als Input für die Frage nach den Textsorten fungieren, die im Zusammenhang mit der Organisation einer Messe, dem Messeauftritt eines Unternehmens und der Vor- und Nachbereitung einer Messe vorkommen. Eine mögliche Projektaufgabe kann z. B. die Suche nach messebezogenen Texten sein, die auf den muttersprachlichen und/oder fremdsprachlichen Internetseiten $\mathrm{zu}$ finden sind, so dass die Kursteilnehmer auf diese Weise ein erstes eigenes Korpus an Paralleltexten erstellen können. Weniger vertraute Textsorten können dann ggf. eingehender und aufgrund der Basisprinzipien der textlinguistischen Analyse untersucht werden (vgl. Brinker 2006: 81 f.; 2005).

1 Im Zusammenhang mit den landesspezifischen Unterschieden zeigte Bernd Spillner (2002: 146 f.), dass die im Deutschen und Französischen übliche Textsorte Zwischenbescheid/réponse (avis) préliminaire, die z. B. im Falle der voraussichtlichen Verzögerung bei der Bearbeitung eines Vorgangs oder Beantwortung eines Schreibens zum guten Ton gehört, in Finnland traditionell nicht bzw. nur selten verwendet wird (zur deutschfinnischen Wirtschaftskommunikation vgl. auch Miebs 2003). 
Die textanalytische Herangehensweise bietet sich besonders bei der Bearbeitung des Themas Bewerbung an. So haben beispielsweise Arbeits-, Zwischen- und Praktikumszeugnisse im Deutschen eine längere Tradition als in manchen anderen Sprachen (z. B. Italienisch) und stellen inzwischen hoch elaborierte Textsorten dar, denen inhaltlich wie formal genau festgelegte Textkonventionen zugrunde liegen. Aus dem Vergleich der Paralleltexte aus der Perspektive der kontrastiven Fachtextologie können darüber hinaus verschiedene kulturell geprägte Formen des Geschäftsumgangs und der Unternehmenskultur erschlossen werden. ( $\mathrm{Zu}$ den Anfängen der kontrastiven Textologie vgl. Spillner 1981.)

Rega bezeichnet die kontrastive Fachtextologie als »eines der zukunftsträchtigsten Forschungsfelder, auch der angewandten Übersetzungswissenschaft« (2006: 91). Im Rahmen ihrer übersetzungsdidaktischen Überlegungen betont sie die Notwendigkeit einer bewussten Auseinandersetzung mit den ausgangs- und zielsprachlichen Textsorten, wobei im Vordergrund diejenigen Textsorten stehen sollen, die für den Übersetzungsmarkt besonders wichtig sind (vgl. ebd.: 93).

Die Entwicklung der Recherchierkompetenz oder allgemeiner des savoir-apprendre, das auf die beschriebene Weise gefördert werden kann, ist für angehende Übersetzer umso wichtiger, als es sich bei der Wirtschaftskommunikation, wie eingangs ausgeführt, um einen der dynamischsten Bereiche der Kommunikation handelt.

\section{Fazit und Ausblick}

Die im Abschnitt 2 betonte Extension des Begriffs Wirtschaft bedeutet, dass die Wirtschaftskommunikation sehr ausdifferenzierte Handlungsfelder und somit auch viele Teil-Fachsprachen und Textsorten umfasst, deren ausschöpfende Klassifikation sich als schwierig oder gar unmöglich gestaltet. (Zu den bisherigen Klassifikationsversuchen vgl. z. B. Hoffmann 1984: 70; Fluck 1977: 33; Frenser 1991: 236 f.; Spillner 2005: 289 f.)

Vielmehr als eine allgemeingültige Klassifikation anzustreben, bietet sich vor diesem Hintergrund an, von der Frage nach dem Zweck des jeweiligen Systematisierungsversuchs auszugehen und die jeweils anzuwendenden Kriterien zielspezifisch zu wählen. So galt in dem hier vorgegebenen Rahmen das Hauptaugenmerk der Frage, welche fachsprachenspezifischen Textsorten Gegenstand des Fachs Wirtschaftsdeutsch für Deutschlernende sein sollen. Es liegt auf der Hand, dass die Lehrwerke für Wirtschaftsdeutsch nur einen fremdsprachendidaktisch vertretbaren Ausschnitt aus der Welt der Wirtschaftskommunikation bieten können und dieser mit der Zeit auch Veränderungen erfahren kann. So stellte z. B. Ursula Frenser vor zwanzig Jahren (1991: 236) fest, dass die wissenschaftliche Wirtschaftsfachsprache für den DaF-Unterricht im Ausland nicht relevant ist und 
das Unterrichtsfach Wirtschaftsdeutsch »mit Berufssprache und landeskundlichfachlichen Informationen über die Wirtschaftsnation Bundesrepublik assoziiert « wird. Dies konnte die hier vorgestellte Lehrwerksanalyse eher für die mittleren Niveaustufen bestätigen: Die B1/B2-Lehrwerke DB3 und UDA liegen vornehmlich im Bereich zwischen allgemeinsprachlich und praxisbezogen-fachsprachlich, weisen weder wirtschaftswissenschaftlichen Anspruch noch eine große Fachwortdichte auf. Anders jedoch die Konzeption des C1-Lehrwerks WKD, in dem auch anspruchsvollere und v. a. wirtschaftswissenschaftlich orientierte Aufgaben ohne direkten Bezug auf die alltägliche Berufspraxis vorkommen und die Tendenz zur Vermittlung wirtschaftswissenschaftlicher Grundkenntnisse und Analysestrategien an mehreren Stellen deutlich erkennbar ist.

Als besonders stark vertreten erwiesen sich in allen drei Lehrwerken explizit adressatenbezogene Texte der externen Unternehmenskommunikation (v. a. Geschäftsbriefe, präsentations- und werbeorientierte Kommunikationsformen), sowie auch - etwa im Unterschied zu den herkömmlichen GeschäftskorrespondenzHandbüchern und zu einigen älteren Lehrwerken - die der internen. Andere Textsorten der Wirtschaftssprache, die nicht unmittelbar auf den Berufsalltag bezogen sind, treten bedeutend seltener auf. So werden z. B. Zeitungstexte zum Thema Wirtschaft, die auch durchaus Lernstoff bieten, eher nur am Rande behandelt oder lediglich in der Lernkontrolle eingesetzt. ${ }^{1}$

Bedeutende Fortschritte zeigen alle drei Lehrwerke im Hinblick auf die grafische Gestaltung: Die dargebotenen Formen der schriftlichen Unternehmenskommunikation wirken im Vergleich zu den älteren in Deutschland veröffentlichten Lehrmaterialien und z.T. auch zu aktuellen französisch- und italienischsprachigen Lehrwerken zur Wirtschaftskommunikation realitätsnäher.

Bei der Textarbeit steht in allen drei Lehrwerken das prozedurale savoir-faire klar im Vordergrund, stellenweise verknüpft mit dem deklarativen Wissen (savoir) in Form von Fach- und Hintergrundwissen. Wie Reuter, Schröder und Tiittula (1991: 111) betonen, besteht die Aufgabe des fachbezogenen Fremdsprachenunterrichts darin, neben dem erforderlichen sprachlichen Wissen und Können auch die erforderliche kommunikative Kompetenz für fremdsprachliche »Handlungsfähigkeit im Fach « zu entwickeln. Die Arbeit an entsprechenden Fertigkeiten ist in den untersuchten Lehrwerken sehr durchdacht und vielfältig, so dass sie in dieser Hinsicht auch manchem allgemeinsprachlichen Lehrwerk als Vorbild dienen könnten. Die in Abschnitt 3 skizzierten Aufgaben sind oft betont entscheidungsorientiert und unterstützen somit auch die Lernerautonomie, die angesichts der Dynamik des Wirtschaftsgeschehens in diesem Bereich geradezu unverzichtbar

${ }^{1}$ Hornung (2008) zeigt in ihrem Vergleich von deutschen und italienischen journalistischen Wirtschaftstexten, dass es auch in diesem Bereich von Sprache zu Sprache sehr unterschiedliche Textkonventionen gibt. 
ist. Wünschenswert im Sinne der Lernerautonomieförderung wären allerdings mehr Hinweise auf lehrwerksexterne Selbstlernmöglichkeiten wie z. B. der Verweis auf die Europass-Internetseite in UDA, die für den Lerner einen ersten Schritt in Richtung selbständiger Beschäftigung mit fachspezifischen Textkonventionen darstellt.

In Bezug auf die Arbeit an fachspezifischen Textmerkmalen kann zusammenfassend Folgendes festgehalten werden: Viele Aufgaben zu den Lesetexten ermöglichen die Bewusstmachung und praktische Umsetzung des fach- und textsortenspezifischen Wortschatzes. Außerdem unterstützen relativ viele Aktivitäten die implizite oder (seltener) explizite Auseinandersetzung mit den Textstruktur- und Textgliederungselementen. Es kommen auch Aufgaben vor, die textsortenspezifische Merkmale, Textfunktion und Textanfänge thematisieren.

Sehr selten sind dagegen Aufgaben, die für kohäsionsstiftende Elemente sensibilisieren (z. B. textsortentypische Konnektoren, Anaphern, Kataphern, Lexemrekurrenz und -variation). Die entsprechenden Aufgabentypen, die beispielsweise in den DSH-Lehrwerken und mehreren em-Ausgaben von Hueber auftreten, fördern nicht nur das Textverstehen, sondern können auch die im Berufsleben durchaus wichtige textproduktive Kompetenz erhöhen.

Eine besonders große Bedeutung im Hinblick auf die produktiven Fertigkeiten kommt z.B. Bewerbungsunterlagen und Geschäftsbriefen zu. Die Analyse der studentischen Arbeiten hat hier einen großen Lern- und Übungsbedarf ergeben, den die untersuchten Lehrwerke nur zum Teil abdecken. Es handelt sich um Textsorten, die zwar syntaktisch und terminologisch gesehen einen eher niedrigen bis mittleren Fachlichkeitsgrad aufweisen, gleichzeitig aber eine hochstandardisierte Makrostruktur haben und die Beachtung vieler länder- bzw. kulturspezifischer Unterschiede voraussetzen (Reinart 2009: 254, 258-262), die von der visuellen Gestaltung bis hin zu den inhaltlichen Bausteinen und ihrer Reihenfolge reichen. Eine intensivere Auseinandersetzung mit den fach- und kulturspezifischen Vertextungsmustern in fachsprachlichen Lehrwerken ist daher als ein Desiderat zu bezeichnen, besonders in den Lernkontexten im Ausland, da die Lerner viel seltener in Kontakt mit authentischen Texten kommen.

Ein weiteres Desiderat wäre eine zielgerichtete und systematische Schulung von interkulturellen Kompetenzen, die den Deutschlernenden neue Perspektiven auf dem internationalen Arbeitsmarkt eröffnen könnten. Reuter beklagt in seinem vor zehn Jahren veröffentlichten Überblick über die Wirtschaftstexte (Reuter 2001: 579), dass Unterrichtsmodelle, die die Außenperspektive und somit die internationale oder die interkulturelle Kommunikation zum Ausgangspunkt des Unterrichts machen, in Deutschland noch nicht ausgearbeitet worden sind. Inzwischen blickt die interkulturelle Kommunikationsforschung auf viele neue Erkenntnisse und praktisch umsetzbare Ergebnisse zurück, die die Lehrwerke allerdings eher illustrativ als systematisch umsetzen. 
Das letzte Untersuchungsziel führt einen Schritt weiter von den fachsprachendidaktischen zu den fachsprachen-übersetzungsdidaktischen Überlegungen. Im Abschnitt 5 wurde aufgezeigt, dass für den Einsatz der drei Lehrwerke im fachsprachlichen Übersetzerunterricht mehrere Gründe sprechen. Die breit gefächerte und für die aktuelle Unternehmenskommunikation repräsentative Textauswahl, die sie (und insbesondere WKD) bieten, kann eine erste Orientierung in der Vielfalt möglicher Kommunikationsformen der Wirtschaft ermöglichen, als Ausgangsbasis bei der eigenständigen Suche nach Paralleltexten dienen und zugleich einen ersten Schritt auf dem Weg zu einer übersetzerrelevanten Klassifizierung bzw. Systematisierung von Textsorten der Wirtschaftskommunikation bilden.

Dabei sind die untersuchten Lehrwerke lediglich als Zusatzquellen und keinesfalls als kurstragende Lehrmittel im Fachübersetzerunterricht zu empfehlen: erstens, weil sie schon allein aus sprachdidaktischen Erwägungen nicht alle Fachsprachen und Textsorten des hochkomplexen Bereichs Wirtschaftsdeutsch darbieten können, und zweitens, weil sie ihren Zielen entsprechend vorrangig nur Textsorten der Unternehmenskommunikation (DB3 und UDA) behandeln, die nicht repräsentativ für den Übersetzungsmarkt und von ungleicher Relevanz für Übersetzer sind (so sind z.B. allgemeine Geschäftsbedingungen, Unternehmensporträt und andere Formen externer Unternehmenskommunikation und -präsentation bedeutend häufiger Bestandteil von Übersetzungsaufträgen als unternehmensinterne Schreiben).

Abschließend sei noch einmal das Potenzial des wirtschaftsbezogenen Deutschunterrichts im Ausland unterstrichen: Er kann die Kommunikation mit den Unternehmen, die (noch) an der deutschen Sprache als Unternehmenssprache festhalten, erleichtern und dadurch indirekt auch die internationale Stellung des Deutschen stärken. Vor diesem Hintergrund erscheint die Reflexion über die optimalen Curricula und Lehrmittel für die Fachfremdsprache Wirtschaftsdeutsch, zu der auch diese Untersuchung einen Beitrag leisten soll, besonders wichtig.

\section{Literatur}

\section{Sekundärliteratur}

Brandt, Andreas von: Deutschland, Italien und Einheit der Nation: ein deutsch-italienischer Blick. Ergebnisse der repräsentativen Meinungsumfrage ISPO/Mannheimer im Auftrag der Deutschen Botschaft Rom, zusammengefasst von Andreas von Brandt. Rom: Deutsche Botschaft Rom, 2011.

Brinker, Klaus: Linguistische Textanalyse. Eine Einführung in Grundbegriffe und Methoden. 6. Auflage. Berlin: Schmidt, 2005.

Brinker, Klaus: »Vorstellung eines textlinguistischen Beschreibungsmodells als Basis des DaF-Unterrichts«. In: Foschi Albert, Marina; Hepp, Marianne; Neuland, Eva (Hrsg.): 
Texte in Sprachforschung und Sprachunterricht. Pisaner Fachtagung 2004 zu neuen Wegen der italienisch-deutschen Kooperation. München: iudicium, 2006, 75-82.

Buhlmann, Rosemarie; Fearns, Anneliese: Handbuch des Fachsprachenunterrichts. Berlin: Langenscheidt, 1987.

Bungarten, Theo (Hrsg.): Aspekte der Unternehmungskultur und Unternehmensidentität in der historischen Wirtschaftslinguistik. Tostedt: Attikon, 1997 (Beiträge zur Wirtschaftskommunikation, 1).

Brünner, Gisela: Wirtschaftskommunikation. Linguistische Analyse ihrer mündlichen Form. Tübingen: Niemeyer, 2000.

Fandrych, Christian; Thurmair, Maria: Textsorten im Deutschen. Linguistische Analysen aus sprachdidaktischer Sicht. Tübingen: Stauffenburg, 2010 (Stauffenburg Linguistik, 57).

Fluck, Hans-Rüdiger: »Fachsprache Wirtschaft - Versuch einer Abgrenzung «. In: Internationales Bulletin der Vereinigung >Sprache und Wirtschaft< 57, 28-51.

Foschi Albert, Marina; Hepp, Marianne; Neuland, Eva (Hrsg.): Texte in Sprachforschung und Sprachunterricht. Pisaner Fachtagung 2004 zu neuen Wegen der italienisch-deutschen Kooperation. München: iudicium, 2006, 75-82.

Frenser, Ursula: »Wirtschaftsdeutsch: ein Verhandlungsgespräch«. In: Müller, BerndDietrich (Hrsg.): Interkulturelle Wirtschaftskommunikation. München: iudicium, 1991, 235243.

GER: Gemeinsamer europäischer Referenzrahmen für Sprachen: Lernen, lehren, beurteilen, Anhang D, Dokument D4: Zusammenfassung der Kann-Beschreibungen im Bereich >Beruf $<$. http:// www.goethe.de/z/50/commeuro/d.htm\#d (Stand: 30.03.2011)

Hoffmann, Lothar: Kommunikationsmittel Fachsprache. Eine Einführung. Berlin: AkademieVerlag, 1984.

Hornung, Antonie: »Mediation - Vermittlung zwischen? Projekt einer interkulturellen Deutschdidaktik im Rahmen der Masterstudiengänge >Unternehmenskommunikation und >Kulturmanagement< Mediazione e trattativa - lingua tedesca«. In: Kaunzner, Ulrike (Hrsg.): Der Fall der Kulturmauer: wie kann Sprachunterricht interkulturell sein? Münster: Waxmann, 2007, 27-41.

Hornung, Antonie: »Tedesco - Il linguaggio specialistico dell'economia nella formazione accademica «. In: Preite, Chiara; Soliman, Luciana T.; Vecchiato, Sara (Hrsg.): Le lingue per gli studenti non specialisti. Nuove strategie di apprendimento/insegnamento. Milano: Egea, 2008, 69-82.

Kautz, Ulrich: Handbuch Didaktik des Übersetzens und Dolmetschens. München: iudicium/ Goethe-Institut, 2002.

Koch, Andreas: »Die historische Wirtschaftslinguistik vor dem Hintergrund der Weltwirtschaftskrise in Deutschland «. In: Bungarten, Theo (Hrsg.): Aspekte der Unternehmungskultur und Unternehmensidentität in der historischen Wirtschaftslinguistik. Tostedt: Attikon, 1997, 32-48 (Beiträge zur Wirtschaftskommunikation, 1).

Kovtyk, Bogdan: »Übersetzung der wirtschaftswissenschaftlichen Texte als sprachliches und extrasprachliches Problem. Beispiel >share«" In: Kovtyk, Bogdan; Wendt, Gabriele (Hrsg): Aktuelle Probleme der angewandten Übersetzungswissenschaft: sprachliche und außersprachliche Faktoren der Fachübersetzung. Berlin u. a.: Lang, 2002, 108-128.

Messing, Ewald E. J.: »Methoden und Ergebnisse der wirtschaftssprachlichen Forschung «. In: Actes du premier congrès international des linguistes à La Haye, 10-15 avril 1928. Leiden: Sijthoff, 1928, 140-142.

Messing, Ewald E. J.: Zur Wirtschafts-Linguistik. Eine Auswahl von kleineren und größeren Beiträgen über Wert und Bedeutung, Erforschung und Unterweisung der Sprache des wirtschaftlichen Verkehrs. Rotterdam: Nijgh \& Van Ditmar N. V., 1932. 
Miebs, Udo: »Höflichkeitssensible Bereiche der finnisch-deutschen Wirtschaftskommunikation und ihre Berücksichtigung in der Sprachschulung «. In: Reuter, Ewald; Piitulainen, Marja-Leena: Internationale Wirtschaftskommunikation auf Deutsch. Die deutsche Sprache im Handel zwischen den nordischen und den deutschsprachigen Ländern. Berlin u. a.: Lang, 2003.

Müller, Bernd-Dietrich (Hrsg.): Interkulturelle Wirtschaftskommunikation. München: iudicium, 1991.

Rega, Lorenza: »Textlinguistische Schwerpunkte in der Übersetzungsdidaktik DeutschItalienisch-Deutsch«. In: Foschi Albert, Marina; Hepp, Marianne; Neuland, Eva (Hrsg): Texte in Sprachforschung und Sprachunterricht. Pisaner Fachtagung 2004 zu neuen Wegen der italienisch-deutschen Kooperation. München: iudicium, 2006, 83-97.

Rega, Lorenza: »Alcune considerazioni linguistico-traduttive sulle Relazioni annuali di Bundesbank, Oesterreichische Nationalbank, Schweizerische Nationalbank / Banca Nazionale Svizzera, Banca d'Italia, Banca Centrale Europea/Europaeische Zentralbank «. In: Schena, Leandro; Preite, Chiara; Soliman, Luciana T.: Le lingue per gli studenti non specialisti. Milano: EGEA, 2008, 123-138.

Reinart, Sylvia: »Professionalität beim Fachübersetzen - am Beispiel von Wirtschaftsfachtexten«. In: Ahrens, Barbara; Cerny, Lothar; Krein-Kühle, Monika; Schreiber, Michael (Hrsg.): Translationswissenschaftliches Kolloquium I, Beiträge zur Übersetzungs- und Dolmetschwissenschaft (Köln/Germersheim). Berlin u. a.: Lang, 2009.

Reuter, Ewald; Schröder, Hartmut; Tiittula, Lisa: »Zur Erforschung von Kulturunterschieden in der internationalen Wirtschaftskommunikation«. In: Müller, Bernd-Dietrich (Hrsg.): Interkulturelle Wirtschaftskommunikation. München: iudicium, 1991, 93-121.

Reuter, Ewald: »Wirtschaftstexte«. In: Helbig, Gerhard; Götze, Lutz; Henrici, Gert; Krumm, Hans-Jürgen (Hrsg.): Deutsch als Fremdsprache Ein internationales Handbuch. Berlin; New York: de Gruyter, 2001, 573-582.

Rocco, Goranka: Deutsch und Deutschlandbild an einer italienischen Universität: Eine Untersuchung zu den Spracheinstellungen der Studierenden. Rom: Aracne editore, 2010 (Lavori interculturali sul Tedesco) (http://www.aperandosini.eu/aperandosini/list_ 3_rocco_it.html)

Schürmann, Gregor R.: »Öffentlichkeitsarbeit als Instrument strategischer Unternehmensführung. Kommunikationsfähigkeit entscheidet über Marktpositionen«. In: Bungarten, Theo (Hrsg.): Selbstdarstellung und Öffentlichkeitsarbeit, Eigenbild und Fremdbild von Unternehmen. Tostedt: Attikon, 1994, 106-113.

Siebenschein, Hugo: Abhandlungen zur Wirtschaftsgermanistik. Prag: Orbis, 1936.

Spillner, Bernd: »Textsorten im Sprachvergleich. Ansätze zu einer Kontrastiven Textologie«. In: Kühlwein, Wolfgang; Thome, Gisela; Wilss, Wolfgang (Hrsg.): Kontrastive Linguistik und Übersetzungswissenschaft. Akten des Internationalen Kolloquiums Trier/Saarbrücken, 25.30.9.1978. München: Fink, 1981, 239-250.

Spillner, Bernd: »Fachtexte im interkulturellen Vergleich. Kontrastive Pragmatik deutscher, finnischer und französischer Wirtschaftstexte«. In: Nuopponen, Anita; Harakka, Terttu; Tatje, Rolf (Hrsg.): Interkulturelle Wirtschaftskommunikation. Forschungsobjekte und Methoden. Vaasa 2002 144-164.

Spillner, Bernd: »Deutsche Wirtschaftssprache: Analyse, Textauswahl, Vermittlung«. In: Heine, Antje; Henning, Mathilde; Tschirner, Erwin (Hrsg.): Deutsch als Fremdsprache. Konturen und Perspektiven eines Faches. Festschrift für Barbara Wotjak zum 65. Geburtstag. München: iudicium, 2005. 


\section{Analysierte Lehrwerke}

Dialog Beruf 3. Kursbuch. Ismaning: Hueber, 2. Auflage 2005.

Dialog Beruf 3. Arbeitsbuch. Ismaning: Hueber, 2. Auflage 2005.

Dialog Beruf 3. Lehrerhandbuch. http://www.hueber.de/shared/elka/Internet_Muster/Red1/ 978-3-19-021592-8_gesamt.pdf

Unternehmen Deutsch Aufbaukurs. Kursbuch. Stuttgart: Klett, 1. Auflage 2007.

Unternehmen Deutsch Aufbaukurs. Arbeitsbuch. Stuttgart: Klett, 1. Auflage 2007.

Unternehmen Deutsch Aufbaukurs. Lehrerhandbuch. Stuttgart: Klett, 1. Auflage 2007.

Wirtschaftskommunikation Deutsch. Berlin: Langenscheidt, 1. Auflage 2008.

Wirtschaftskommunikation Deutsch. Lehrerhandreichungen. http://www.langenscheidt-unterrichtsportal.de/lehrerhandreichungen_754.html

\section{Goranka Rocco}

Geb. 1971; Dr. phil.; Studium der Germanistik und Romanistik an den Universitäten Zagreb und Essen und zweijähriges Zusatzstudium der schwedischen Sprache, Promotion 2003 in Romanistik und Germanistik an der Universität Düsseldorf, 1996 bis 2006 DaF- und DaZ-Dozentin an den Universitäten Duisburg und Essen und an mehreren Sprachinstituten, 2003-2006 wissenschaftliche Angestellte an der Universität Düsseldorf, seit 2006 DAAD-Lektorin an der Universität Bologna. Forschungsschwerpunkte: kontrastive Fachtexttypologie, Fachsprachenforschung, Übersetzungswissenschaft, Fachsprachen- und Übersetzungsdidaktik, Soziolinguistik, Spracheinstellungen, Migrationsliteratur. 